\title{
Dislocation model for strain accumulation in a plate collision zone
}

\author{
Kunihiko Shimazaki and Yanlai Zhao \\ Earthquake Research Institute, University of Tokyo, Yayoi 1-1-1, Bunkyo-ku, Tokyo 113-0032, Japan
}

(Received December 31, 1999; Revised June 19, 2000; Accepted July 6, 2000)

\begin{abstract}
Following a scheme developed for a subduction zone by Savage in 1983, which was successfully applied to a transform plate boundary by Matsu'ura and others in 1986, a dislocation model for a plate collision zone is formulated. The solution consists of a rigid plate motion and a tensile dislocation. In addition to this, a strike-slip dislocation is needed when the collision boundary is not perpendicular to the plate converging direction. Theoretically predicted gradual change in horizontal displacements over the collision zone well explains the results of GPS continuous observation in central Japan where the Eurasian and Okhotsk plates are thought to be colliding. The maximum uplift rate is predicted as $1 / \pi$ times that of converging velocity, however the observed uplift rate is much smaller than that, although vertical movements observed by GPS network is much less accurate than horizontal movements. A comparison of the theoretical results with the observation suggests relatively thin elastic plates, whose thickness is about $30 \mathrm{~km}$. The obtained dislocation model has a close connection with a horizontal detachment fault. The displacement fields above the advancing and retreating edges of a horizontal rectangular detachment fault are mathematically equivalent to those of collision and rift zones, respectively, and lateral edges to a transform fault boundary. The displacement fields at a colliding and a rifting boundaries are the same except for their signs.
\end{abstract}

\section{Introduction}

A dislocation model of interseismic deformation in a subduction zone is formulated by Yamashina (1976) and Savage (1983) and the same scheme is applied to a transform-fault plate boundary (Savage and Burford, 1973; Matsu'ura et al., 1986). Although the observed crustal deformations along the Pacific coastal areas of Japan have been successfully modeled by using Savage's formulations (e.g. Yoshioka et al., 1993; Shen-Tu and Holt, 1996; El-Fiky and Kato, 1999a), those in the central part of Japan where the Okhotsk and Eurasian plates are thought to be colliding (Seno et al., 1996) has not been well explained. In this study a dislocation model for strain accumulation process caused by the plate collision is proposed following Savage (1983)'s derivation. The proposed model includes a dislocation solution for a tensile crack, which was included in Brown (1975)'s attempt for general formulation of plate interaction. In this paper, the derivation of the dislocation model is first described. Then its relation with a horizontal detachment fault and other plate boundaries will be discussed, together with the nature of the dislocation model. Finally, an application of the model to the colliding plate boundary in central Japan will be shown.

\section{Dislocation Model}

The strain accumulation process in a collision zone can be expressed as a superposition of two processes as was done by Savage (1983) for a subduction zone. Figure 1 shows this superposition model. The top illustration shows strain accumulation process at a collision zone. The middle one

Copy right (C) The Society of Geomagnetism and Earth, Planetary and Space Sciences (SGEPSS); The Seismological Society of Japan; The Volcanological Society of Japan; The Geodetic Society of Japan; The Japanese Society for Planetary Sciences. represents rigid plate motions. It includes an imaginary process of removing an excess mass to accommodate the plate convergence. This imaginary mass removal has to be compensated and needs a supplemental solution, which is a dislocation model of tensile crack shown at the bottom. The amount of tensile dislocation is equal to the amount of plate convergence.

When a plate converging vector is not perpendicular to the colliding boundary, not only a tensile crack but also a strikeslip dislocation is necessary. The vector can be decomposed into two components: one perpendicular to the boundary and the other parallel to it. The perpendicular component can be treated as was described above by a tensile-crack dislocation. The parallel component can be treated in a similar way to the model proposed for a transform fault (Matsu'ura et al., 1986).

It should be noted here that the above arguments also apply to a strain accumulation process on a rifting boundary. Instead of a tensile crack, anti-tensile crack has to be introduced, and the plate divergence rate should be used in place of the convergence rate.

In Fig. 1, a vertical boundary for the plate collision is shown. This is because any dipping boundary is found to be equivalent to the vertical boundary (Zhao, 1999). The essential point is the bottom of the plate boundary, where flows in the asthenosphere converge as shown by the arrows. Thus we take a point on the earth's surface just above this point of convergence as the origin of the coordinates. When the boundary is dipping, the horizontal plate convergence vector can be decomposed into two: one perpendicular to the boundary and the other parallel to it. The perpendicular component can be treated in a similar way to that described above. Instead of a vertical tensile crack, a dipping tensile crack has to be used in this case. The parallel component can 


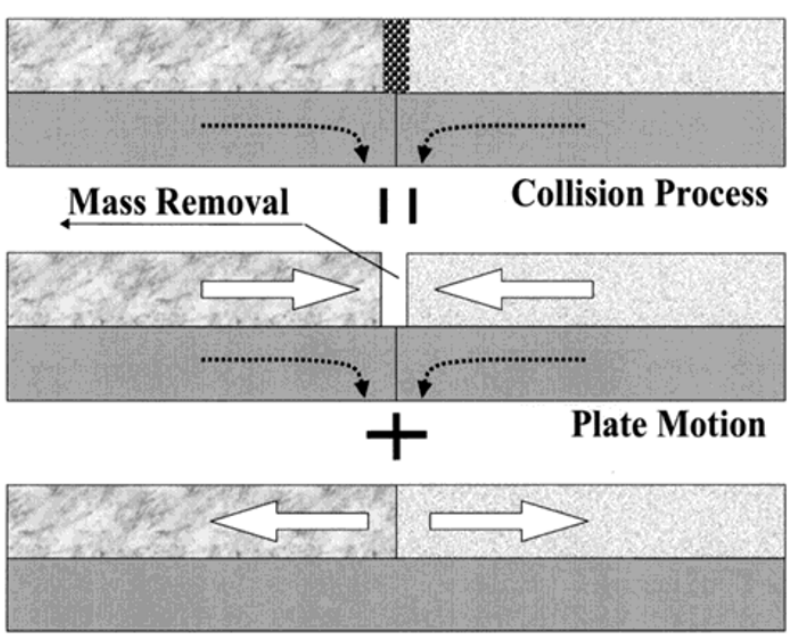

Tension Crack

Fig. 1. Superposition model of strain accumulation at a collision zone following Savage (1983)'s scheme. Removal of excess mass is necessary to maintain a rigid plate motion, which is compensated by a tensile crack dislocation.

be treated in the same fashion as was described by Savage (1983) by a normal fault. The final result can be obtained by a superposition of the dipping tensile crack and the dipping normal fault, which turns out to be equivalent to the vertical tensile crack. This was numerically confirmed by using Okada (1985)'s code.

For a case of two dimensions, i.e. an infinitely long colliding plate boundary, the horizontal and vertical displacements, $U_{x}$ and $U_{z}$ are given by the followings (Zhao, 1999):

$$
\begin{aligned}
U_{x} & =\frac{U}{\pi}\left(\frac{x H}{x^{2}+H^{2}}-\tan ^{-1} \frac{x}{H}+\frac{\pi}{2}\right) \\
U_{z} & =\frac{U}{\pi} \frac{H^{2}}{x^{2}+H^{2}}
\end{aligned}
$$

where $U$ and $H$ represent the amount of plate convergence and the thickness of the plates, respectively. We assume $U_{x}=U_{z}=0$ at $x=\infty$. These formulae can be derived by using the scheme shown in Fig. 1 and the closed form representation of dislocation obtained by Okada (1985). The normalized horizontal and vertical displacements, i.e., $U_{x} / U$ and $U_{z} / U$ are shown in Fig. 2. In this figure, the horizontal distance is normalized by the plate thickness which is the only scale parameter included in the above equations. The dominant wave length of the displacement fields is much longer than the scale parameter. The wave length roughly amounts to eight times the plate thickness.

The obtained dislocation model of strain accumulation at a colliding plate boundary has a close connection with the strain accumulation mechanism due to a horizontal detachment fault. Iio (1996), Ikeda (1992), and Hirahara et al. (1998) proposed that strain accumulation in the Japanese islands away from the trench axis is caused by a detachment fault. Figure 3 shows a map view of a rectangular horizontal detachment fault. Displacements near the four edges can be calculated by using Okada (1985)'s formulae. When effects of other edges can be neglected, i.e., in a case of two dimensions, the displacement fields above each edge turn out to

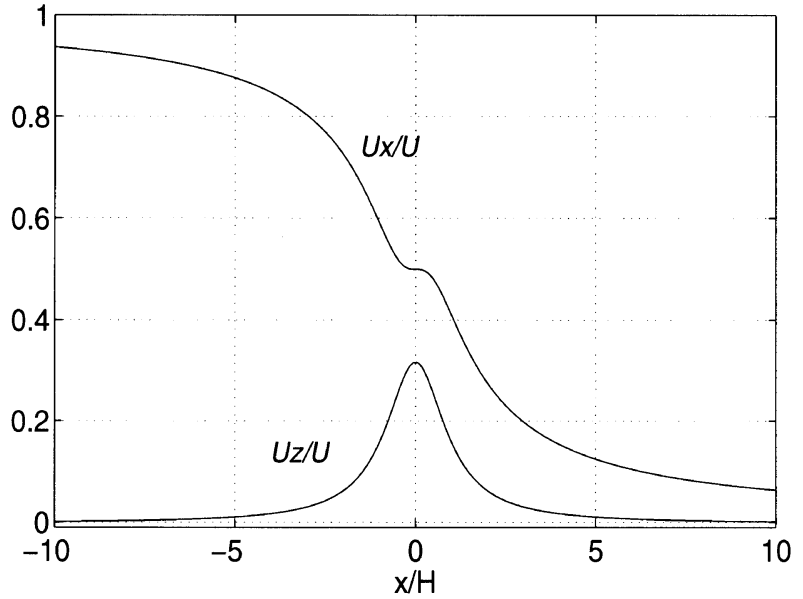

Fig. 2. Normalized horizontal and vertical surface displacements due to strain accumulation at a collisional plate boundary. The origin of the $x$-coordinate is at the point of plate convergence. We assume $U_{x}=U_{z}=0$ at $x=\infty$.

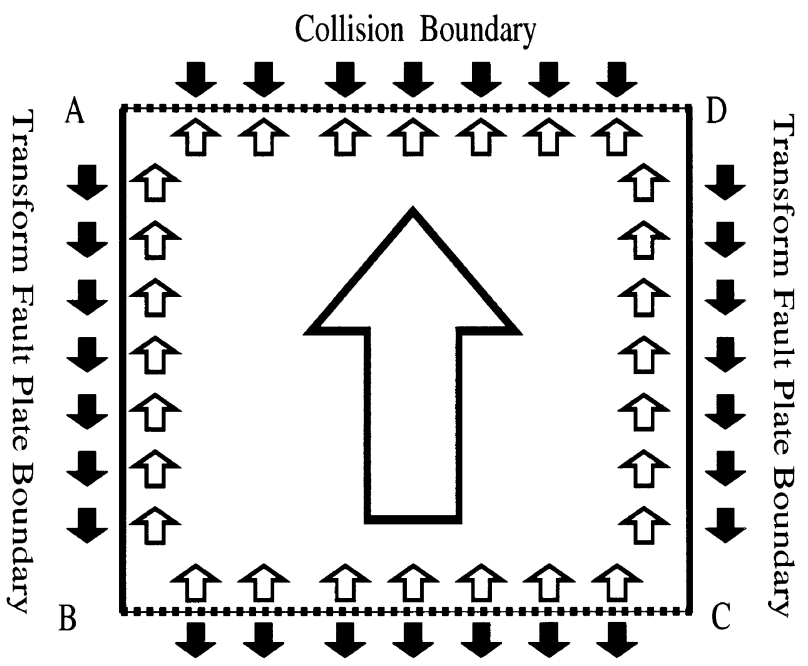

Rift Zone Plate Boundary

Fig. 3. Rectangular horizontal detachment fault (map view).

be mathematically equivalent to those of a plate boundary formulated in this study and by Savage and Burford (1973): advancing and retreating edges correspond to a colliding and a rifting plate boundaries, respectively, and lateral edges to a transform fault boundary (Zhao, 1999). Thus the same displacement field can be interpreted by both the collision model and the detachment fault. When the detachment fault is proposed, however, an existence of the other three edges is usually neglected.

\section{GPS Results}

In central Japan, the Eurasian and the Okhotsk plates are thought to be colliding at a rate of $1.3 \mathrm{~cm} / \mathrm{yr}$ in a direction of $\mathrm{N} 100^{\circ} \mathrm{E}$ (Seno et al., 1996). It could be argued that other plates are colliding at this boundary and this point will be discussed later in this paper. According to Seno et al. (1996) the boundary runs along the Itoigawa-Shizuoka tectonic line. 
However, Tada (1996) and Tada et al. (1997) proposed on the basis of the strain field estimated from the repeated triangulation surveys and of the velocity field obtained from GPS observation, respectively, that the boundary in the northern part runs more than tens of kilometers east of the tectonic line, along a series of faults forming the western boundary of the Nagano basin. Further Zhao (1999) suggested on the basis of GPS observation, the boundary in the southern part may be branching into two, one along the tectonic line and the other lying west of it and striking WSW.

Figure 4 shows smoothed horizontal velocity field in central Japan (Zhao, 1999) deduced from GEONET (GPS Earth Observation Network) results (Miyazaki et al., 1997, 1998) provided by the Geographical Survey Institute of Japan. It is spatially filtered for noise reduction by a Gaussian function with a correlation distance of $100 \mathrm{~km}$ following the least square collocation method developed by El-Fiky and Kato (1999b) on the basis of Moritz (1962)'s work on gravity. Yasato station which is located within the Okhotsk plate and shown by the solid triangle in the figure was assumed to be fixed. This station may be moving westward due to a subduction of the Pacific plate, but its effect would be smaller than several millimeters per year judging from the distance from the axis of the Japan trench where the Pacific plate is subducting beneath the Okhotsk plate.

Figure 4 shows the dominant eastward velocity field which shows the motion of the Eurasian plate relative to the Okhotsk plate, except for the northern and southern Pacific coasts where the effect of subduction of the Pacific and the Philippine Sea plates, respectively, is dominant. Since some complication in plate boundary is suggested in the southern part, the observed velocity field in the northern part is compared with that calculated from the proposed dislocation

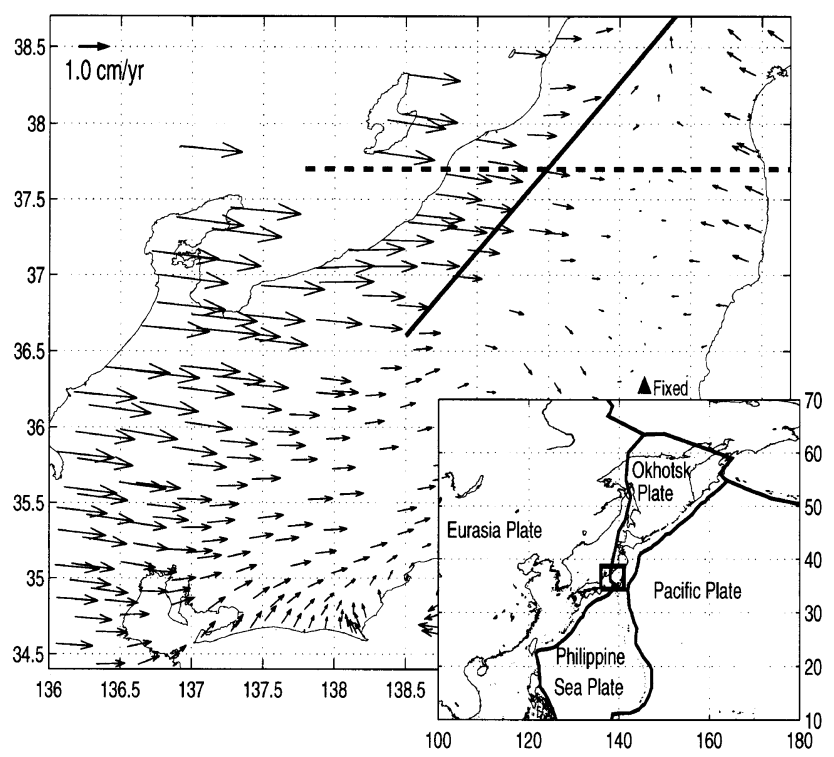

Fig. 4. Velocity field in central Japan deduced from GPS observation (GEONET) data (Zhao, 1999). The solid triangle indicates Yasato station which is assumed to be fixed. The solid line shows the assumed colliding boundary and the broken line shows $37.7^{\circ} \mathrm{N}$ latitude line where the theoretical velocity fields shown in Fig. 5 were calculated. The inset shows plate boundaries in the northwestern Pacific region. model. The strike of the collision boundary shown by the solid line and the direction of convergence are assumed to be $\mathrm{N} 40^{\circ} \mathrm{E}$ and $\mathrm{E}-\mathrm{W}$, respectively. Also the plate convergence rate is assumed to be $2.5 \mathrm{~cm} / \mathrm{yr}$, which is much larger than that estimated from Seno et al. (1996)'s plate model.

Eastward horizontal and vertical velocities were calculated at all the GPS stations on the basis of the proposed model. Then we applied the same filter as was used for the observed data, i.e., the Gaussian filter with a correlation distance of $100 \mathrm{~km}$, to the calculated velocities for a direct comparison of the observation with the model. Figure 5 shows the comparison on latitude lines, $37.5^{\circ}, 37.6^{\circ}, 37.7^{\circ}, 37.8^{\circ}, 37.9^{\circ}$, and $38.0^{\circ} \mathrm{N}$. Since the predicted velocity fields are similar, examples calculated on $37.7^{\circ} \mathrm{N}$ latitude line (shown by the broken line in Fig. 4) are shown in Fig. 5. Although the theoretical velocity fields were spatially smoothed, the difference between the cases of plate thicknesses of 7 and $15 \mathrm{~km}$ is discernable. In general theoretical velocity field calculated for a plate thickness of $30 \mathrm{~km}$ well explains the observed fields. The eastern part of the observed horizontal velocities (distance $>50 \mathrm{~km}$ in Fig. 5) has to be neglected because it is apparently affected by the subduction of the Pacific plate. In the area of comparison (distance $\leq 50 \mathrm{~km}$ ), the effect of the subduction of the Pacific plate can be evaluated as a few millimeters per year or less (Ito et al., 2000).

The maximum calculated vertical velocity appears to be smaller than $1 / \pi$ times that of the assumed plate convergence rate. This is caused by the oblique convergence and the
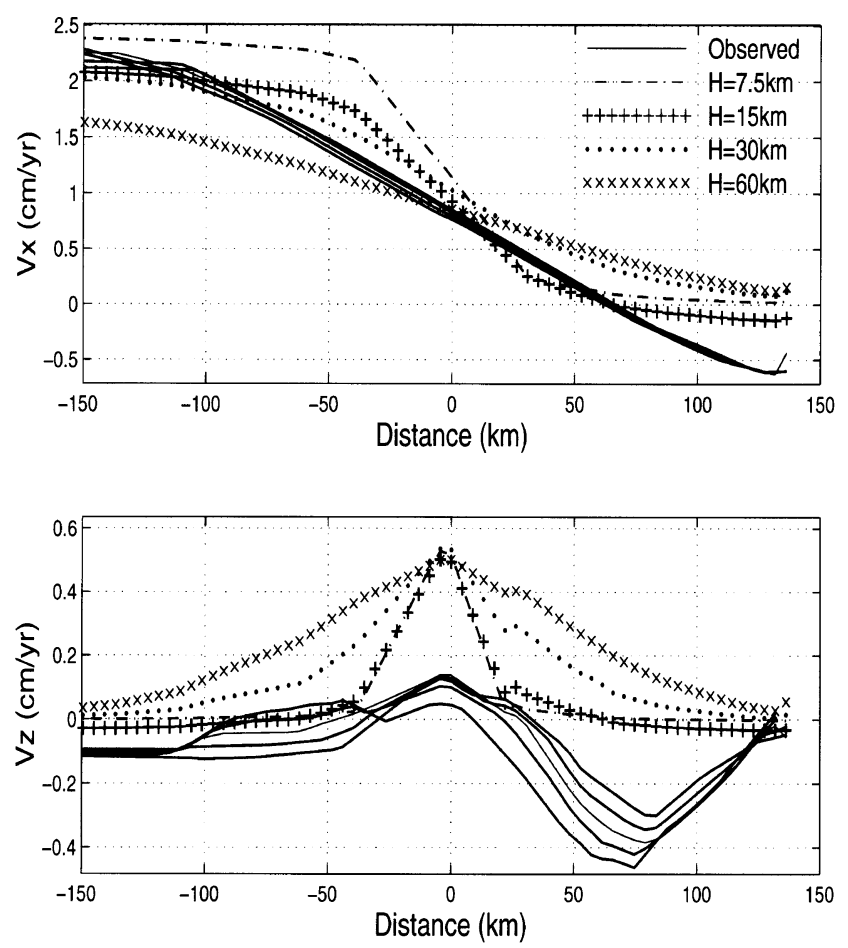

Fig. 5. Comparison of the observed with the theoretical velocity fields Eastward horizontal velocities are shown above and vertical velocities are shown below. The thin lines show the observed velocity fields on $37.5^{\circ}$, $37.6^{\circ}, 37.7^{\circ}, 37.8^{\circ}, 37.9^{\circ}$, and $38.0^{\circ} \mathrm{N}$ latitude line. The theoretical velocity fields are calculated on $37.7^{\circ} \mathrm{N}$ latitude line shown by the broken line in Fig. 4 for various thickness of the plate. 
spatial filtering. The observed vertical velocities are smaller than those calculated from the model and they become flat and take a value of $-0.1 \mathrm{~cm} / \mathrm{yr}$ about $110-150 \mathrm{~km}$ west of the boundary, which may suggest that the reference station is being uplifted at a rate of $0.1 \mathrm{~cm} / \mathrm{yr}$. If it is the case, the observed uplift rate is about half that of the model.

\section{Discussion and Conclusion}

The proposed dislocation model produces the horizontal velocity field which well explains the observation in the northern part of central Japan, where the two plates are thought to be colliding. However, the observed vertical velocity is much smaller than the theoretical one. The difference in velocity, a few millimeters per year does not seem to be critical because it is smaller than the estimated accuracy in vertical velocity obtained from GPS observation (Zhao, 1999).

The assumed plate convergence rate is about twice that calculated from Seno et al. (1996)'s plate model. However, there are other plate models: Wei and Seno (1998) proposed that the west of this boundary lies the Amurian plate instead of the Eurasian plate, and Heki et al. (1999) argued that on this boundary the Amurian and the North American plates are colliding. The proposed convergence rates are $1.5 \mathrm{~cm} / \mathrm{yr}$ (Wei and Seno, 1998) and $1.8 \mathrm{~cm} / \mathrm{yr}$ (Heki et al., 1999). If the fixed station, Yasato is moving westward at a rate of several millimeters per year, and if Heki et al. (1999)'s estimate is correct, the observed velocity field is very consistent with the dislocation model within the uncertainty in observation not only on its pattern but also on its absolute value. An alternative argument would be that the observed velocity field includes a significant amount of uniform crustal shortening (Ikeda, personal communication). If this is the case, the estimated rate of $2.5 \mathrm{~cm} / \mathrm{yr}$ representing the plate convergence velocity includes the effect of both the plate collision and the uniform shortening.

In this study, a dislocation model for the plate collision zone is formulated, which consists of a rigid plate motion and a tensile dislocation. In addition to this, a strike-slip dislocation is needed when the collision boundary is not perpendicular to the plate converging direction. Theoretically predicted velocity field over the collision zone well explains the results of GPS continuous observation in the northern part of central Japan. A comparison of the theoretical results with the observation suggests relatively thin elastic plates, whose thickness is $30 \mathrm{~km}$. The displacement field at a colliding boundary is the same as that on a rifting boundary except for their signs.

Acknowledgments. We thank Dr. H. Kawawa and Ms. Y. Kikuta at the Geographical Survey Institute who kindly provided us with a large amount of GPS data. We also thank to Prof. M. Matsu'ura at the University of Tokyo, for giving us constructive critical comments on our dislocation model in the early stage of this work and Prof. K. Hirahara at Nagoya University for his encouragements, and Prof. Y. Ikeda at the University of Tokyo and an anonymous reviewer for their helpful reviews.

\section{References}

Brown, R. J., A dislocation approach to plate interaction, Ph.D. Thesis, Massachusetts Institute of Technology, 1975.

El-Fiky, G. S. and T. Kato, Interplate coupling in the Tohoku district, Japan, deduced from geodetic data inversion, J. Geophys. Res., 104, 2036120377, 1999a.

El-Fiky, G. S. and T. Kato, Continuous distribution of the horizontal strain in the Tohoku district, Japan, predicted by least-square collocation, Geodynamics, 27, 213-236, 1999b.

Heki, K., S. Miyazaki, H. Takahashi, M. Kasahara, F. Kimata, S. Miura, N. F. Vasilenko, A. Ivashchenko, and K. D. An, The Amurian plate motion and current plate kinematics in eastern Asia, J. Geophys. Res., 104, 2914729155, 1999.

Hirahara, K., M. Ando, Y. Hoso, Y. Wada, and T. Nakano, Local GPS array for the movement of the Atotsugawa fault, Chikyu, 20, 149-153, 1998.

Iio, Y., A possible generating process of the 1995 southern Hyogo prefecture earthquake-stick of fault and slip on detachment—, J. Seismol. Soc. Japan, 49, 103-112, 1996.

Ikeda, Y., Reverse faults of Japan: On the possibility of flake tectonics, Chikyu-Gogai, 5, 137-152, 1992.

Ito, T., S. Yoshioka, and S. Miyazaki, Interplate coupling in northeast Japan deduced from inversion analysis of GPS data, Earth Planet. Sci. Lett., 176, 117-130, 2000 .

Matsu'ura, M., D. D. Jackson, and A. Cheng, Dislocation model for aseismic crustal deformation at Hollister, California, J. Geophys. Res., 91, 1266112674, 1986.

Miyazaki, S., T. Saito, M. Sasaki, Y. Hatanaka, and Y. Iimura, Expansion of GSI's nationwide GPS arrays, Bull. Geogr. Surv. Inst., 43, 23-34, 1997.

Miyazaki, S., T. Saito, Y. Hatanaka, T. Sagiya, and T. Tada, The nationwide GPS array as an Earth Observation System, Bull. Geogr. Surv. Inst., 44, 11-22, 1998.

Moritz, H., Interpolation and prediction of gravity and their accuracy, Rep. 24, Inst. Geod. Photogr. Cartogr., Ohio State Univ., Columbus, U.S.A., 1962

Okada, Y., Surface deformation due to shear and tensile faults in a half-space, Bull. Seismol. Soc. Am., 75, 1135-1154, 1985.

Savage, J. C., A dislocation model for strain accumulation and release at a subduction zone, J. Geophys. Res., 88, 4984-4996, 1983.

Savage, J. C. and R. O. Burford, Geodetic determination of relative plate motion in central California, J. Geophys. Res., 78, 832-845, 1973.

Seno, T., T. Sakurai, and S. Stein, Can the Okhotsk plate be discriminated from the North American plate?, J. Geophys. Res., 101, 11305-11315, 1996.

Shen-Tu, B. and W. E. Holt, Interseismic horizontal deformation in northern Honshu and its relationship with the subduction of the Pacific plate in the Japan trench, Geophys. Res. Lett., 23, 3103-3106, 1996.

Tada, T., Seismotectonic provinces in the Japanese islands based on the horizontal strain of the earth's crust, Chikyu, 18, 807-811, 1996.

Tada, T., T. Sagiya, and S. Miyazaki, Deforming Japanese islands as seen from GPS observations, Kagaku, 67, 917-927, 1997.

Wei, D. and T. Seno, Determination of the Amurian plate motion, in Mantle Dynamics and Plate Interactions in East Asia, edited by M. Flower et al. Geodynamics Ser., 27, pp. 337-346, 1998.

Yamashina, K., Drag model and crustal deformation in the Japanese islands, Program Abstr., Seismol. Soc. Japan, 1, 56, 1976.

Yoshioka, S., T. Yabuki, T. Sagiya, T. Tada, and M. Matsu'ura, Interplate coupling and relative plate motion in the Tokai district, central Japan, deduced from geodetic data inversion using ABIC, Geophys. J. Int., 113, 607-621, 1993.

Zhao, Y., Application of dislocation model for plate collision to aseismic crustal deformation in central Japan, Ph.D. Thesis, University of Tokyo, 1999

K. Shimazaki (e-mail: nikosh@eri.u-tokyo.ac.jp) and Y. Zhao (e-mail zhaoyl@eri.u-tokyo.ac.jp) 